\title{
Biomarkers in the assessment of oral mucositis in head and neck cancer patients: a systematic review and meta-analysis
}

\author{
Ana Gabriela Costa Normando ${ }^{1}$ • Camila Lopes Rocha ${ }^{2}$ • Isabela Porto de Toledo ${ }^{1,3}$. \\ Paulo Tadeu de Souza Figueiredo ${ }^{1}$ - Paula Elaine Diniz dos Reis ${ }^{1}$ • \\ Graziela De Luca Canto $^{3,4}$ • Eliete Neves Silva Guerra ${ }^{1}$
}

Received: 23 December 2016/Accepted: 7 June 2017 /Published online: 16 June 2017

(C) Springer-Verlag GmbH Germany 2017

\begin{abstract}
Purpose The aim of this study was to evaluate the capability of biomarkers to predict the risk of oral mucositis in head and neck cancer patients, as well as to assess the correlation between these biomarkers and the severity of mucositis.

Methods The search was performed at LILACS, PubMed, Science Direct, Scopus, and Web of Science. A search of the gray literature was performed on Google Scholar, OpenGrey, and ProQuest. The methodological quality of the included studies was assessed using the Meta-Analysis of Statistics Assessment and Review Instrument (MAStARI) tool, and the evidence quality was assessed by the Grading of Recommendation, Assessment, Development, and Evaluation (GRADE) system.

Results After a two-step selection process, 26 studies met the eligibility criteria. In total, 27 biomarkers were evaluated, and the most frequent were the epidermal growth factor (EGF), Creactive protein (CRP), genetic polymorphisms, tumor necrosis factor alpha (TNF- $\alpha)$, and erythrocyte sedimentation rate (ESR). The meta-analysis showed an expression of
\end{abstract}

Electronic supplementary material The online version of this article (doi:10.1007/s00520-017-3783-8) contains supplementary material, which is available to authorized users.

Eliete Neves Silva Guerra

elieteneves@unb.br

1 Health Sciences Faculty, University of Brasília, Brasília, DF, Brazil

2 Faculty of Dentistry, Federal University of Ceará, Fortaleza, Brazil

3 Brazilian Centre for Evidence-Based Research, Department of Dentistry, Federal University of Santa Catarina, Florianópolis, Brazil

4 School of Dentistry, Faculty of Medicine and Dentistry, University of Alberta, Edmonton, Canada polymorphisms in XRCC1 (32.66\%), XRCC3 (31.00\%), and RAD51 (39.16\%) genes, as well as an expression of protein biomarkers $(39.57 \%)$, in patients with an increased risk of developing oral mucositis.

Conclusions Dosing biomarkers before starting radiation therapy may be a promising method to predict the risk of developing mucositis and allow radiosensitive patients to have a customized treatment. Although there is currently limited evidence to confirm the putative implementation of serum and salivary biomarkers to assess the correlation between them and the severity of mucositis, this current review provides new research directions.

Keywords Biological markers · Oral mucositis · Head and neck cancer $\cdot$ Radiotherapy $\cdot$ Systematic review $\cdot$

Meta-analysis

\section{Introduction}

Lip, oral cavity, and pharyngeal cancers are head and neck cancer (HNC) subside that have been estimated to be responsible for 529,500 incident cases and 292,300 deaths in 2012, accounting for about $3.8 \%$ of all cancer cases and $3.6 \%$ of cancer deaths [1]. The main objective of the treatment for HNC, which is performed by surgery, radiation therapy (RT), and chemoradiotherapy (CRT), is to maximize the probability of cure, while minimizes the risks of toxicity that compromise patient's function and quality of life [2]. Many complications can arise from the nonsurgical therapy of cancer, and the most common are mucositis, dermatitis, taste alteration, xerostomia, dysphagia, anorexia, and fatigue [2, 3]. Oral mucositis $(\mathrm{OM})$ is an inflammatory response of the oropharyngeal mucosa due to cancer therapy, and it is considered 
one of the most significant of all side effects in the head and neck region. It is usually a limiting factor for the intensification of therapy, and it may lead to a break in the treatment, compromising its efficacy [2-6]. OM is characterized by erythema, ulceration, swelling, and pain. It has different levels of severity that vary between patients and can be more severe when there is an association of chemotherapy (CT) and RT [5, $7,8]$. OM is a painful condition that affects patient's quality of life because it impairs the ability to eat, to swallow, and to talk, which increases the costs because it requires hospitalizations due to necessity of pain control, use of parenteral nutrition, and infection management $[4,6,9]$. Mucositis grade may vary according to the dose of treatment, size of irradiated area, and fractioning planning and seems to be regulated by many molecules, for example epidermal growth factor (EGF) and tumor necrosis alpha (TNF- $\alpha$ ), supporting that mucositis is not just an epithelial process $[5,10]$.

Heterogeneity in the response to RT in normal tissues is observed among patients who are treated with identical doses of radiation. Comprehending the molecular details of the pathogenesis of $\mathrm{OM}$ allows the early identification of patients prone to develop severe OM, as well as facilitate the monitoring and characterization of this adverse effect [11]. Clinical studies have been made in order to obtain a biomarker capable to predict the risk of the patient, so that protocols of individual treatments can be planned $[3,11]$. A biomarker is any structure, substance secreted by the tumor, metabolic pathway, or process that can be employed for diagnosis, prognosis, and prediction of pathogenic processes or pharmacological responses to a therapeutic intervention, which can be measured accurately and reproducibly [12]. Proteins are the main biomarkers, and they can be detected in fluids, like blood and saliva, or body tissues [13]. In this way, knowing that a patient has an elevated risk of developing OM will help to plan a customized intervention with curative surgery, peripheral RT, or low-dose CT, to prevent the development of the condition [9].

Thus, the purpose of this systematic review and metaanalysis was to identify biomarkers for the prediction of risk of oral mucositis in head and neck cancer patients, as well as to assess the correlation between these biomarkers and the severity of mucositis.

\section{Methods}

This systematic review reported accordingly the Preferred Reporting Items for Systematic Reviews and Meta-Analyses (PRISMA) checklist [14]. The protocol was registered at the International Prospective Register of Systematic Reviews (PROSPERO) database under registration number CRD42016037299 [15].

\section{Study design}

A systematic review of human studies was undertaken to evaluate the capability of biomarkers to predict the risk of occurrence of $\mathrm{OM}$ and to assess the severity of $\mathrm{OM}$ in $\mathrm{HNC}$ patients.

\section{Eligibility criteria}

\section{Inclusion criteria}

Articles that evaluated biomarkers of patients with HNC undergoing RT or CRT to predict the risk of occurrence of OM or to assess the severity of OM related to cancer treatment were reviewed. Only patients under RT or CRT were included because according to the NCCN guidelines for treatment of newly diagnosed $\mathrm{HNC}$, the standard therapy is based on the performance status of the patient and RT is always in the treatment protocol, whether as a definitive therapy, associated with systemic therapy, or as palliative treatment [16].

\section{Exclusion criteria}

Studies were excluded for the following reasons: (1) studies that evaluated gastrointestinal mucosa; (2) patients with other types of cancer, different from HNC; (3) data not individualized for HNC; (4) only chemotherapy was used to cancer treatment; (5) no correlation between biomarkers and severity/risk of development of OM; (6) reviews, letters, personal opinions, book chapters, and conference abstracts; (7) association between biomarkers and $\mathrm{OM}$ in experimental studies (clinical trials, in vitro or in vivo animal studies); and (8) language restrictions.

\section{Information sources and search strategy}

Studies to be considered for inclusion were identified using an individual search strategy for each of the following electronic databases: LILACS, PubMed, Science Direct, Scopus, and Web of Science (Online Resource 1). A partial gray literature search was performed using Google Scholar, OpenGrey, and ProQuest Dissertations \& Theses Global. The search across the databases included all articles published up to the 25th of January 2016, and the gray literature search included all articles published up to the 1st of February 2016, with no time restriction. Duplicated references were removed by reference manager software (EndNote ${ }^{\circledR}$, Thomson Reuters). In addition, the reference lists of selected articles were hand screened for potential relevant studies that could have been missed during the electronic database searches. 


\section{Study selection}

The study selection was completed in two phases. In phase 1, two authors (AGCN and CLR) independently reviewed titles and abstracts identified in all electronic databases and selected articles that appeared to meet the inclusion criteria. In phase 2, the same authors (AGCN and CLR) independently read the full text of all selected articles and excluded studies that did not meet the inclusion criteria (Online Resource 2). Disagreements between the two initial evaluators were solved by consensus. When they did not reach a consensus, a third reviewer (ENSG) was involved to make a final decision. Reference lists for all included articles were critically assessed by AGCN. The articles that were selected from the reference lists were read by AGCN and CLR.

\section{Data collection process}

One author (AGCN) collected key information from each selected article. A second reviewer (CLR) cross-checked the collected information and confirmed its accuracy. Again, any disagreement between them was resolved by discussion and mutual agreement among AGCN, CLR, and ENSG. For all of the included studies, the following information was recorded: year of publication, author(s), country, sample size (cases of HNC and non-HNC controls), patient age, drug dose, type and class of biomarkers, study methods, type of study, and main conclusions.

\section{Risk of bias in individual studies}

The risk of bias of selected studies was evaluated using the standardized critical appraisal instrument for risk of bias assessed by the Meta-Analysis of Statistics Assessment and Review Instrument (MAStARI) critical appraisal tools [17]. Risk of bias was categorized as high when the study reaches up to $49 \%$ score "yes," moderate when the study reached 50 to $69 \%$ score "yes," and low when the study reached more than 70\% score "yes." AGCN and CLR scored each item as "yes," "no," "unclear," or "not applicable" and assessed independently the quality of each included study (Online Resource 3). Disagreements were resolved by a third reviewer (IPT).

\section{Summary measures}

The primary outcome for this systematic review was the capacity of biomarkers to predict the risk of occurrence of OM in patients with HNC undergoing RT or CRT. A secondary outcome was the capability of biomarkers to assess the severity of $\mathrm{OM}$ in HNC patients. Any type of outcome measurement was considered in this review (categorical and continuous variables).

\section{Synthesis of results}

Proportion meta-analysis of polymorphisms and protein expression associated to the risk of developing OM was performed using MedCalc statistical software, version 14.8.1 (MedCalc Software, Ostend, Belgium), based on values of subjects from the total sample and from cases where the polymorphisms or proteins were expressed. Heterogeneity was calculated by inconsistency indexes $\left(I^{2}\right)$, and a value greater than $50 \%$ was considered an indicator of substantial heterogeneity between studies [18]. The significance level was set at $5 \%$.

\section{Risk of bias across studies}

Clinical heterogeneity (by comparing variability among the participant's characteristics and outcomes studied), methodological heterogeneity (by comparing the variability in study design and risk of bias), and statistical heterogeneity were considered.

\section{Confidence in cumulative evidence}

The Grading of Recommendation, Assessment, Development, and Evaluation (GRADE) instrument [19, 20] assessed evidence quality and grading of recommendation strength in the 26 studies included in qualitative synthesis. This assessment was based on study design, risk of bias, inconsistency, indirectness, imprecision, and other considerations. Evidence quality was characterized as high, moderate, low, or very low [19, 20]. The GRADE was assessed using the website http://gradepro.org.

\section{Results}

\section{Study selection}

In phase 1 of the study selection, 1028 citations were identified across the five electronic databases. After the duplicate articles were removed, 893 citations remained. Comprehensive evaluation of the titles and abstracts was completed, and 857 articles were excluded, so 36 articles were selected after phase 1 . The search with Google Scholar yielded 296 references, of which only one was included for full-text analysis and included in data collection. Six additional articles were identified from the reference lists of the identified studies, but only two of them were included in the analysis. Twenty articles were identified using ProQuest and four articles were identified using OpenGrey, but none of these studies were included.

A full-text review was conducted on the 43 articles retrieved from phase 1 of the selection. This process led to the 
exclusion of 17 studies [21-37]. At the end, 26 articles were selected for descriptive analysis [3, 11, 38-61]. A flow chart detailing the process of identification, inclusion, and exclusion of studies is shown in Fig. 1.

\section{Study characteristics}

The studies were conducted in 16 different countries: Belgium [60], Brazil [48], Canada [46, 55], China [52], Finland [53, 58], Germany [11, 47, 49, 50], Greece [61], India [3, 38, 41], Iran [57], Israel [54], Italy [56], Korea [51], Sweden [44], Taiwan [39, 40], UK [59], and USA [42, 43, 45]. All 26 studies were published between 1994 and 2015, three of them were conducted before the 2000s, and the other 23 studies were published after 2000. One study [37] was excluded because it was written in Chinese, and the authors could not analyze it. Thus, all of the included studies were published in English.

The total sample from the 26 selected studies included 1007 individuals affected by HNC. Sample size ranged from 10 [45] to 183 [3] HNC patients. All of the included studies evaluated patients undergoing RT, but some studies appraised patients who underwent both RT and CRT.

Different biological factors were detected in different samples such as saliva, blood, and tissues. Sixteen studies (62\%) evaluated serum biomarkers, seven studies (27\%) appraised salivary biomarkers, and the three remaining studies (11\%) evaluated the biomarkers on biopsy specimens and cytological smears.

A summary of the descriptive characteristics for the included studies that assessed serum, salivary, and tissue biomarkers is presented in Tables 1,2, and 3, respectively.

\section{Risk of bias within studies}

The summary of risk of bias assessment of the 26 included studies is presented in Table 4. Seven studies were graded as a moderate risk of bias, while the other 19 were considered as a low risk of bias. In item 2 of the MAStARI methodological quality criteria, the patients were only considered to be at a similar point in the course of their condition if all of them were exposed to the same treatment (only RT or only CRT). Item 3 (Has bias been minimized in relation to selection of cases and of controls?) was applicable only for the studies with case and control groups included in the present review [41, 43, 48, 59]. Item 5 (Are the outcomes assessed using objective criteria?) was entirely scored as "yes" because in all studies, the measurement tools used were validated instruments. The majority of the studies had a follow-up over a sufficient time period, measured the outcomes in a reliable way, and used appropriate statistical analysis. On the other hand, most studies did not identify confounding factors nor described the outcomes of people who withdrew.

\section{Results of individual studies}

Despite heterogeneity among the types of biomarkers evaluated, many studies concluded that the biomarkers tested had the capability to correlate with the severity of OM in HNC patients $[11,38-42,44-46,48-51,54,55,61]$ or predict the risk of occurrence of OM $[3,11,46,56,60]$. Although many studies had as main purpose finding an association between biomarkers and the appearance of mucositis, the conclusions of the studies also indicated a possible correlation before treatment. In this way, a patient who has, prior to radiotherapy, a high level of a certain biomarker that has already been known to be overexpressed in patients with severe OM is more likely to develop mucositis during the treatment.

\section{Synthesis of results}

In total, 27 biomarkers were assessed in the included studies (Fig. 2). To easily interpret the results, the biomarkers were grouped in eight different groups: growth factors, acute-phase inflammatory markers, genetic factors, cytokines, general proteins, plasma antioxidants, apoptotic proteins, and cells (Fig. 3). The most frequent types of biomarkers were growth factors, other inflammatory markers, and genetic factors (Online Resource 4).

Among the growth factors, stood out the EGF and transforming growth factor beta 1 (TGF- $\beta 1$ ) that were appraised in seven of the 26 studies. It was observed that there was a trend of reduced EGF levels in patients with severe OM, corroborating the hypothesis that patients with lower levels of EGF prior to therapy may be at increased risk of mucosal damage during RT [43, 45, 46], although Citrin et al. [42] could not find a variation in the concentration of EGF $(p=0.0001)$. Differently, the TGF- $\beta 1$ levels seemed to be elevated if the radiation toxicity was severe $[39,40]$, while Lundberg et al. [53] could not find a significant correlation between the severity of mucositis and the TGF- $\beta 1$ genotype $(p=0.25)$.

The most frequent acute-phase inflammatory markers analyzed were C-reactive protein (CRP) and erythrocyte sedimentation rate (ESR), and three studies $[41,51,55]$ evaluated both markers, since the two are related to acute phase response. The studies demonstrated a significant increase in CRP and ESR levels towards the end of RT and found a correlation between those high levels and fraction number and grade of mucositis. Only Ki et al. [51] could not find any relationship between ESR levels and the fraction number or the grade of mucositis $(p=0.58)$.

The other biomarker that was very frequent among the studies was the genetic polymorphisms, which were analyzed in three different studies $[3,56,60]$. Higher chances of developing acute toxicities like $\mathrm{OM}$ were reported to be associated to polymorphisms in the XRCC1 $(p=0.011)$, XRCC3 $(p=0.178)$, and $\operatorname{RAD51}(p=0.728)$ genes. 


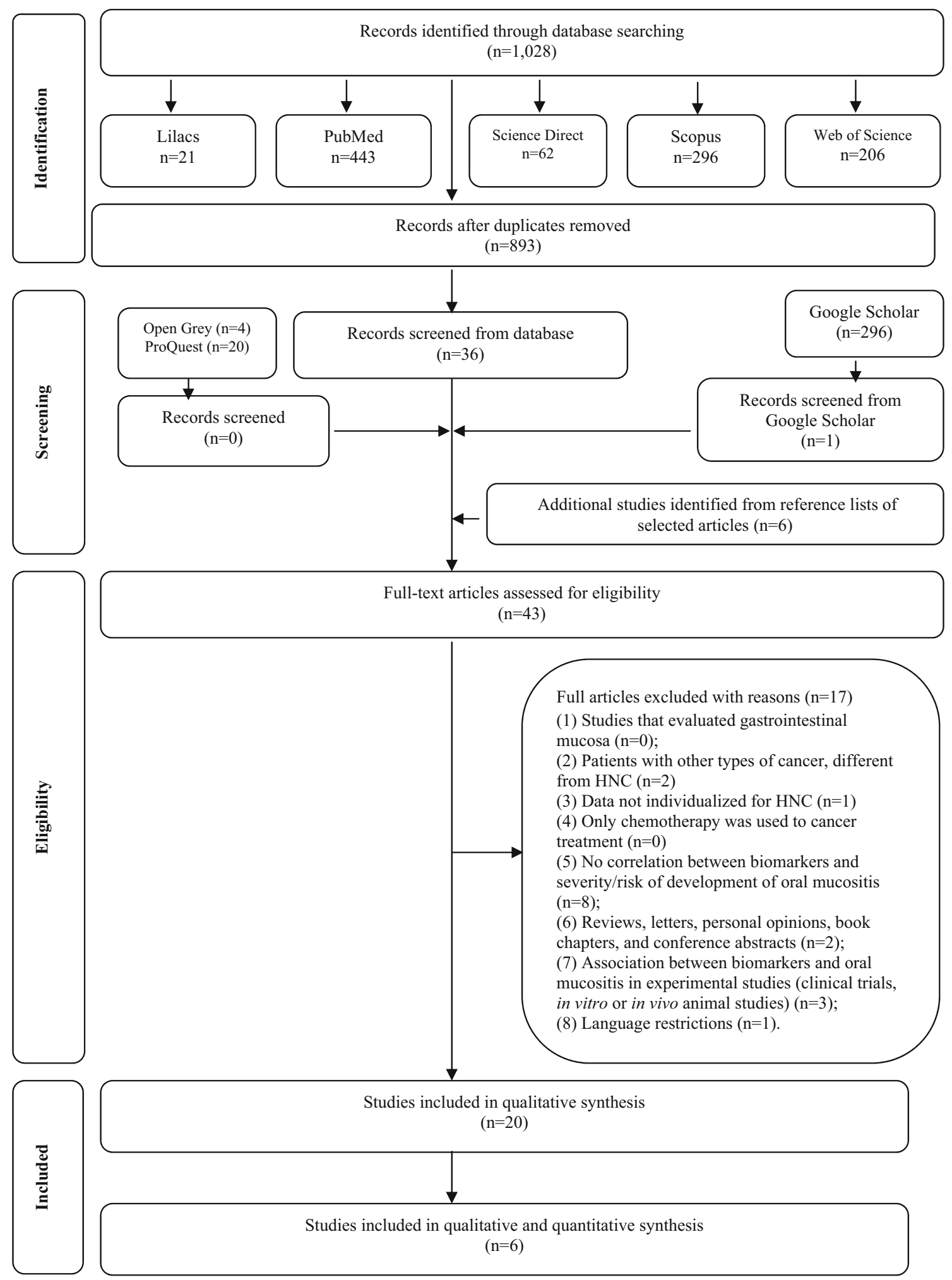

Fig. 1 Flow diagram of literature search and selection criteria adapted from PRISMA [15]

The cytokines evaluated in the included studies were IL-1, IL-1 $\beta$, IL-4, IL-6, IL-8, IL-10, and TNF- $\alpha$. The levels of IL- 6 , IL- 8 , IL-10, and IL- $1 \beta$ increased according to radiation dose, and only IL- 8 did not seem to be related to severe OM $[42,54,57,61]$. TNF- $\alpha$ levels seemed to be increased in some studies [42,61] but decreased in two other studies [54,57], indicating a contradiction in the results. 


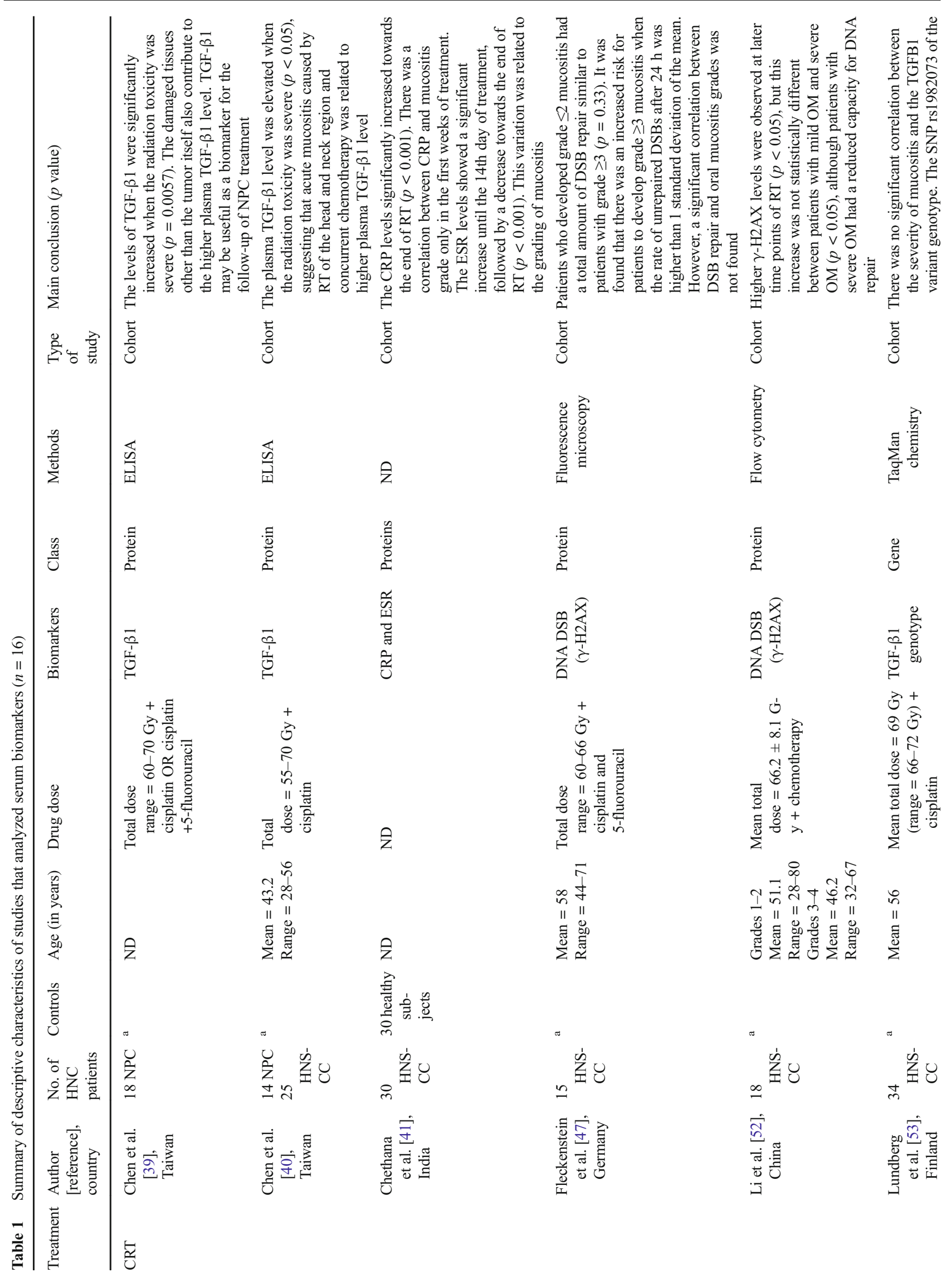




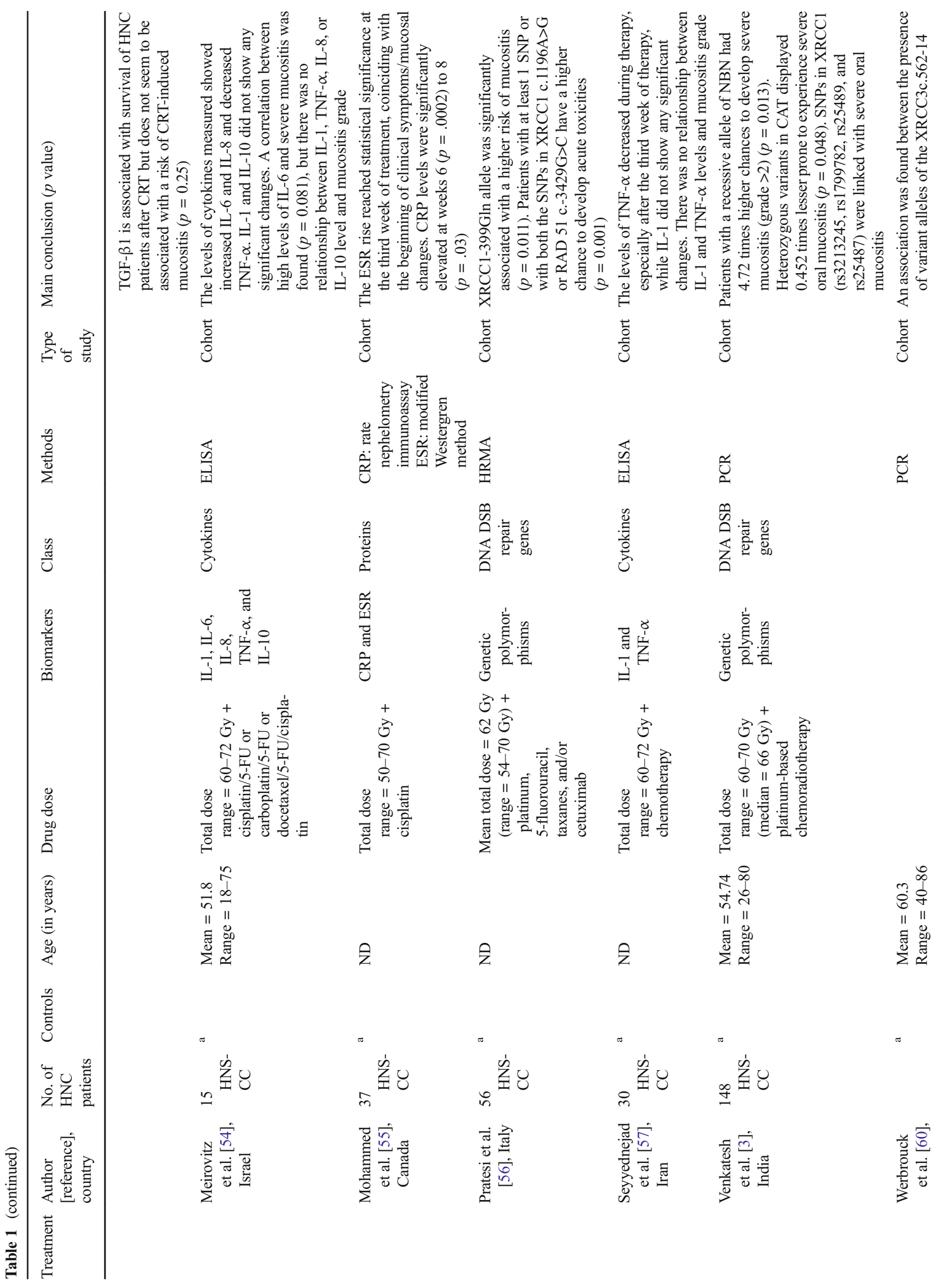




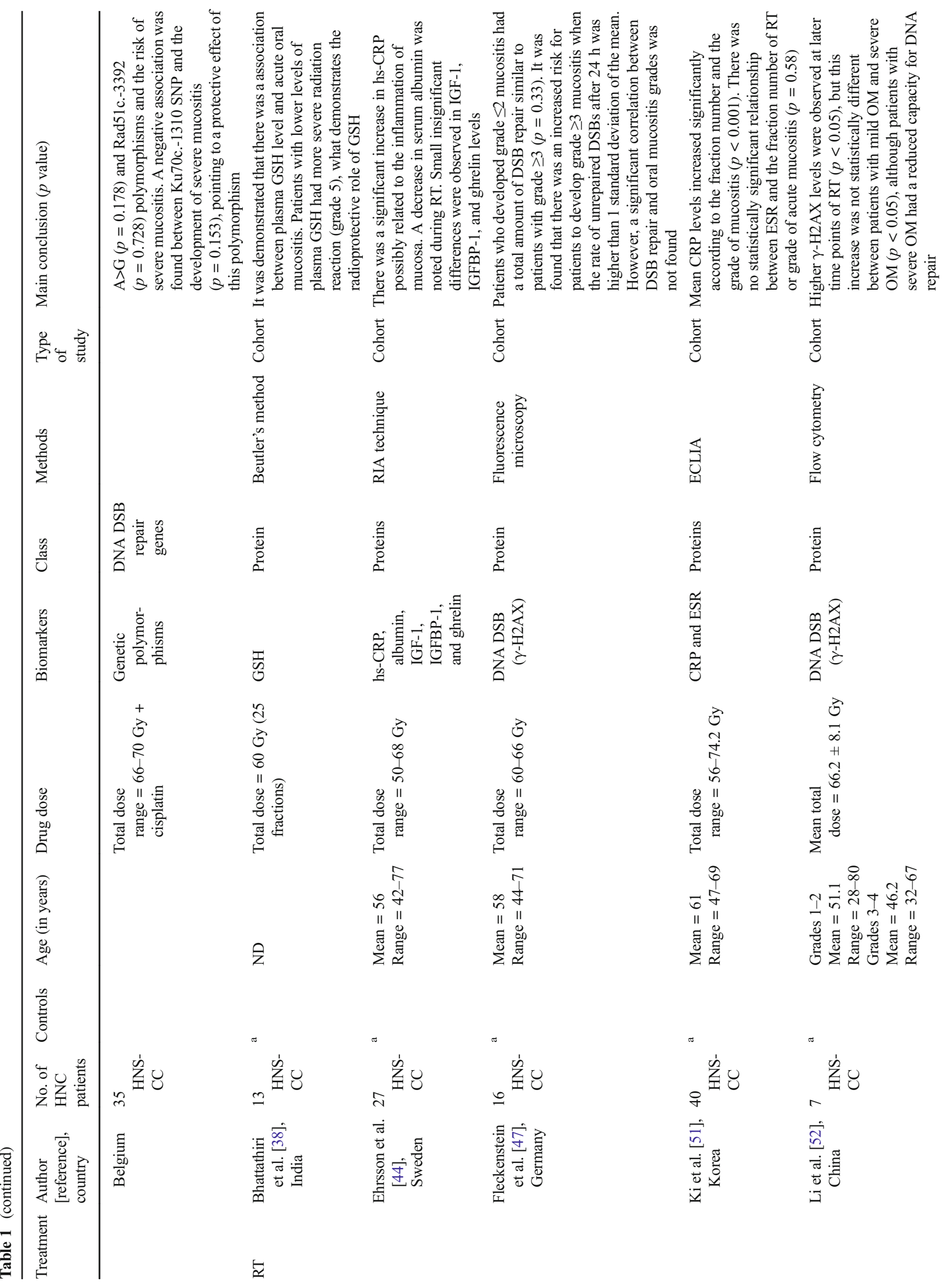




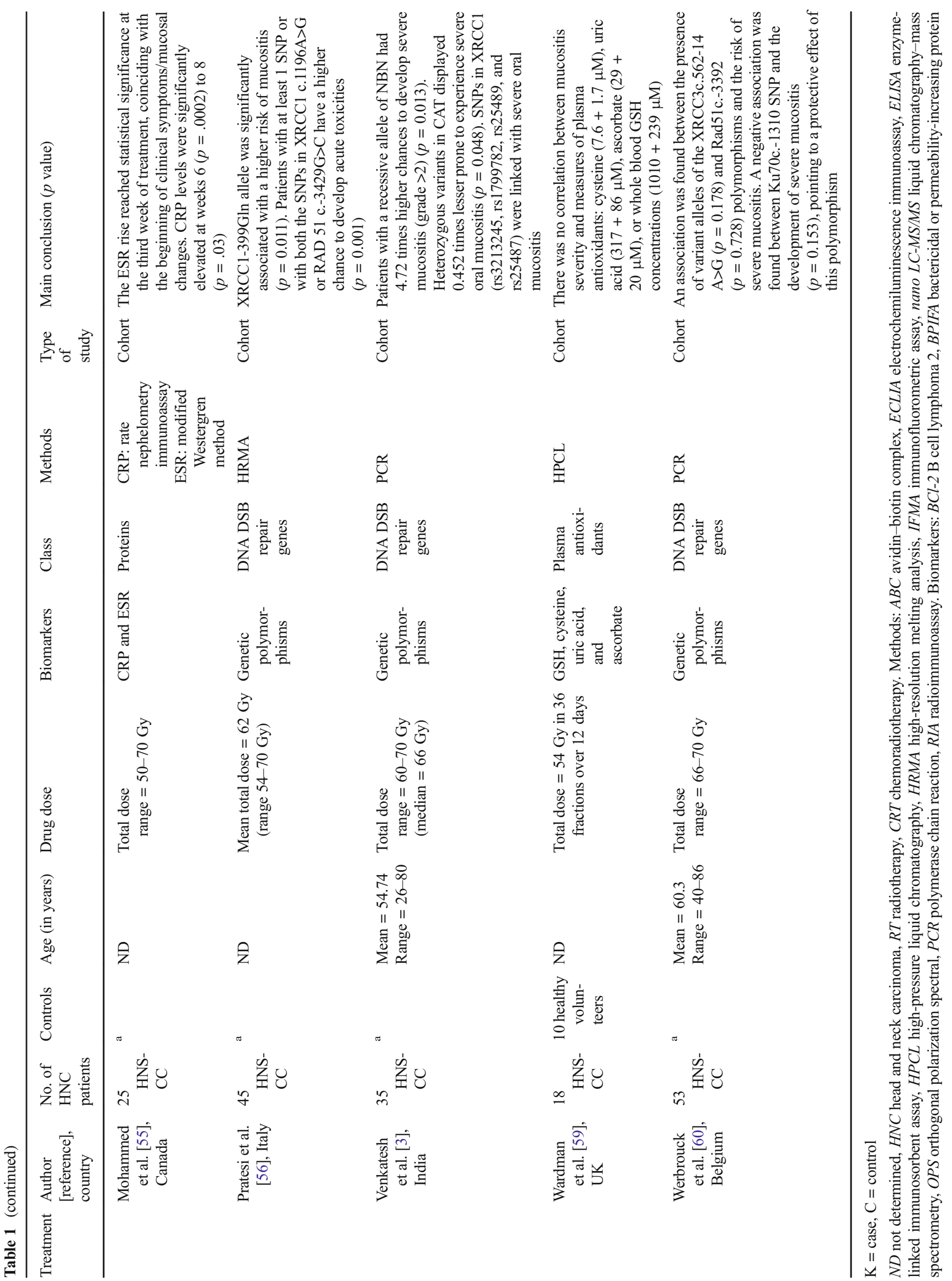


Six studies had enough data to be included in the quantitative synthesis, i.e., the number of patients who developed OM and expressed the biomarker was provided, and were suitable for grouping for meta-analysis. The high heterogeneity between the studies was found in all meta-analyses. For polymorphisms in XRCC1 (rs25487), an inconsistency $\left(I^{2}\right)$ of $90.93 \%$ [confidence interval (CI) 83.02-95.16) was found; for the polymorphisms in XRCC3 (rs861539), $I^{2}$ was 96.72\% (CI 94.78-97.93); for the polymorphisms in RAD51 (rs1801321), $I^{2}$ was 95.22\% (CI 93.07-96.70); and for the expression of protein biomarkers, an inconsistency $\left(I^{2}\right)$ of $53.19 \%$ (CI 4.21-77.12) was found. Accordingly, the random model was chosen. Results from the meta-analysis showed a frequency (prevalence) from the overall expression of XRCC1 polymorphism of $32.66 \%$ (CI $21.52-44.90, p<0.0001$, $n=663$ ), from the expression of XRCC3 polymorphism of $31.00 \%$ (CI 13.84-51.44, $p<0.0001, n=663$ ), from the expression of RAD51 polymorphism of $39.16 \%$ (CI 26.66$52.44, p<0.0001, n=1116$ ), and from the overall expression of protein biomarkers of 39.57\% (CI 28.03-51.73, $p=0.0233$, $n=146$ ) (Fig. 4a-d). Supplementary data from all metaanalyses can be found in Online Resource 5.

\section{Risk of bias across studies}

The included studies used similar methodology, which reduced the possibility of misinterpretation. All studies selected were considered to be relatively homogeneous, since all of them were observational studies. Besides this particular issue, in the meta-analysis, high heterogeneity was found in the selected studies possible due to the sample size that varied widely among the studies.

\section{Quality of evidence}

Overall, the quality of the evidence from the outcomes evaluated by the GRADE system was assessed as moderate, suggesting a moderate confidence in the estimated effect, but there is a possibility that it is substantially different (Online Resource 6).

\section{Discussion}

\section{Summary of evidence}

The possibility of measuring the risk of developing $\mathrm{OM}$ in HNC patients that underwent RT may improve the management of such condition and may allow patients' customized treatment strategies that prevent severe toxicities $[42,52]$. The biomarkers can be considered promising tools for this purpose. This is the first systematic review and meta-analysis that investigated in the available literature whether biomarkers can predict the risk of developing OM in patients with $\mathrm{HNC}$ 


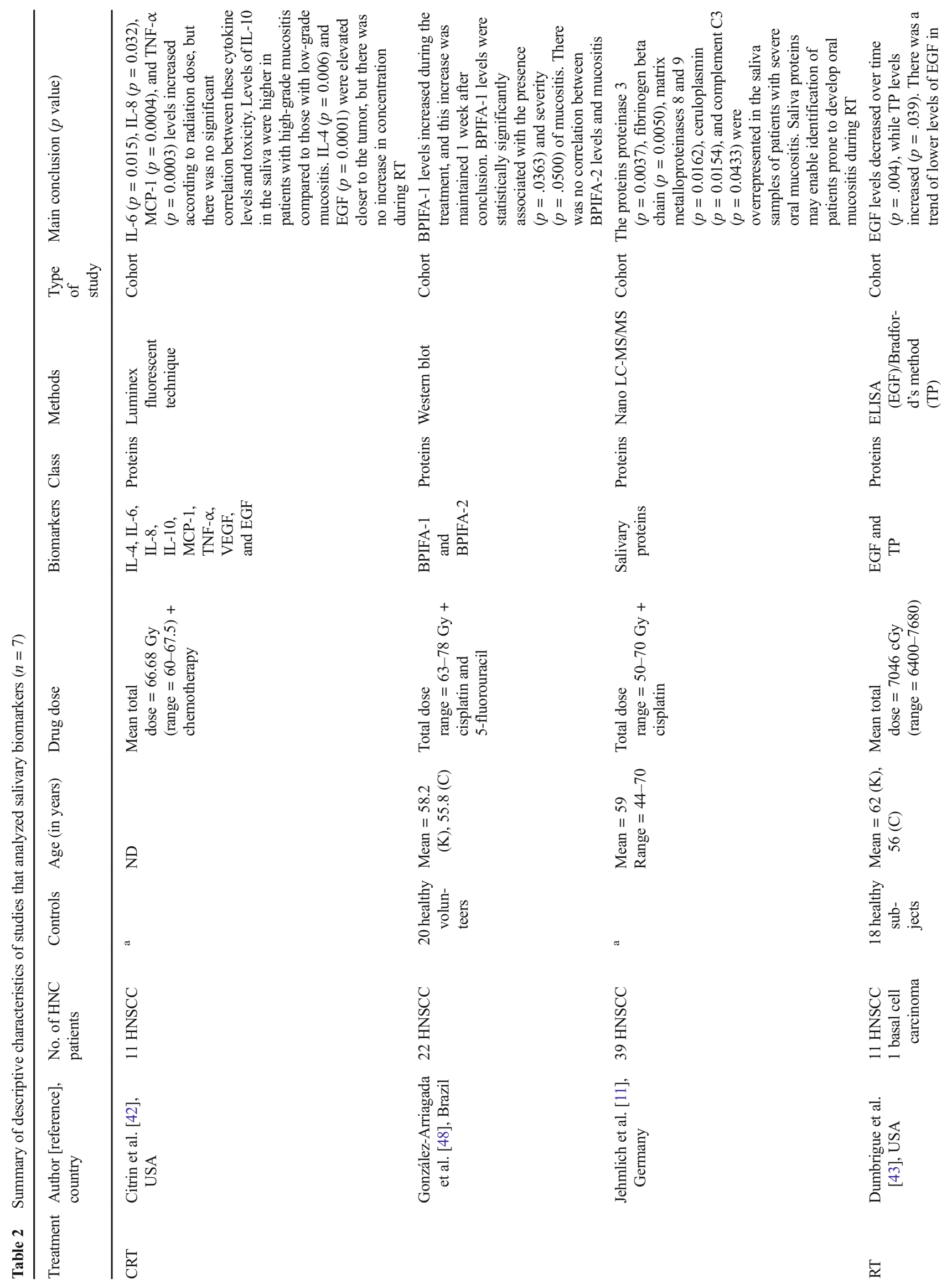




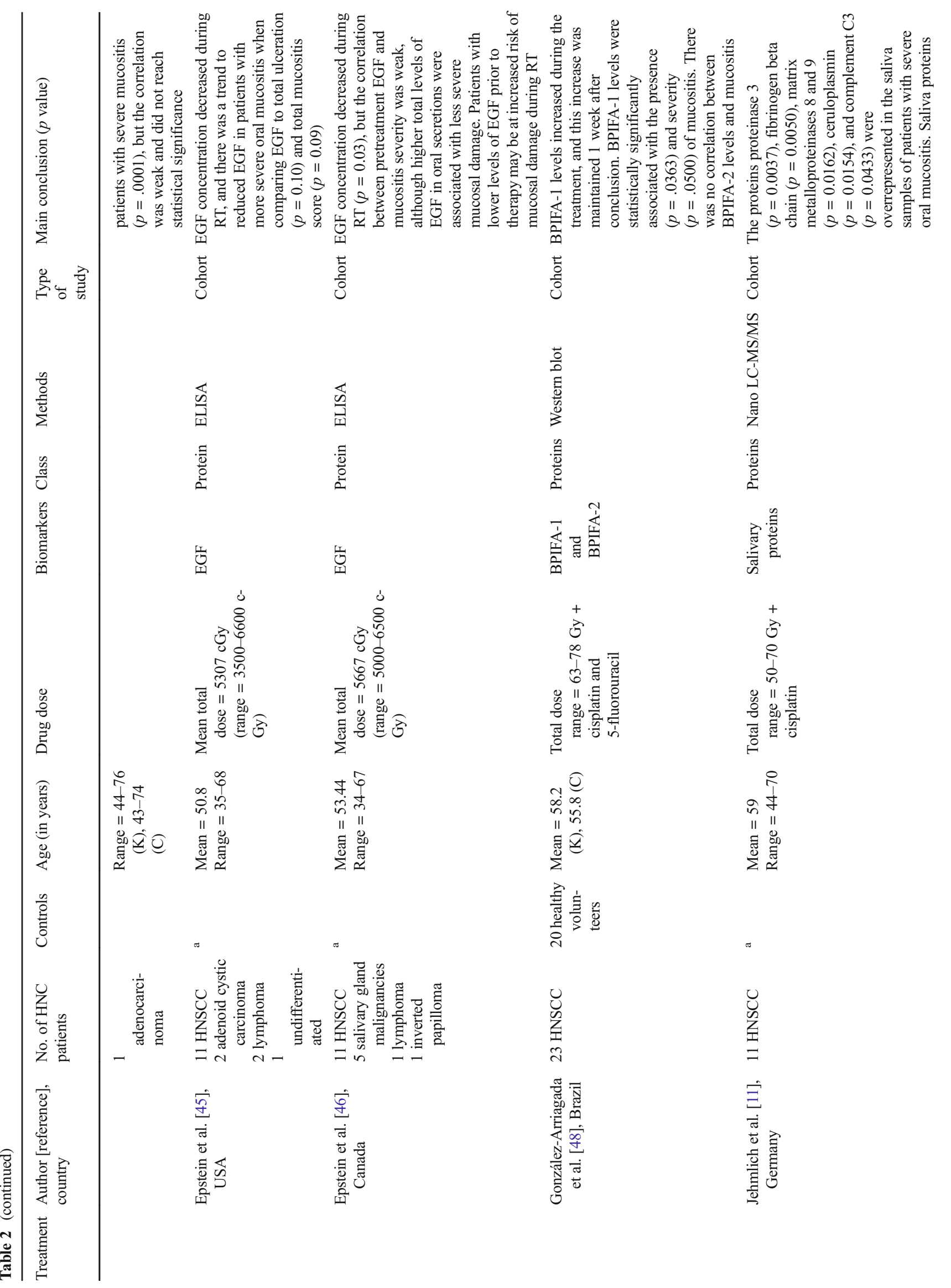


undergoing RT or CRT. Eight groups of biomarkers were analyzed: growth factors, cytokines, acute-phase inflammatory markers, genetic factors, general proteins, plasma antioxidants, apoptotic proteins, and cells.

Growth factors are proteins released by individual cells to transmit messages to other cells and to stimulate cellular growth, proliferation, and differentiation [63]. Regarding the EGF, three studies $[43,45,46]$ observed a decrease in EGF levels during RT and a trend to reduced EGF in patients with more severe OM. These findings suggest that patients with lower levels of EGF prior to therapy may be at increased risk of mucosal damage during RT. Thus, analyzing EGF levels before starting the RT could be an efficient method to identify patients with a higher risk of developing oral mucositis.

Another important growth factor analyzed was the TGF- $\beta$, which controls cellular homeostasis and proliferation, wound healing, immunosuppression, and angiogenesis [53]. It was observed that a TGF- $\beta 1$ level was significantly higher in patients experiencing severe radiation toxicity, confirming that damaged tissues contribute to higher plasma TGF- $\beta 1$ level $[39,40]$. Furthermore, the production of TGF- $\beta 1$ is genetically regulated and patients who have the variant allele at the single nucleotide polymorphism (SNP) in the TGFB1 gene tend to have a higher concentration of serum TGF- $\beta 1$ [53]. However, Lundberg et al. [53] could not find a significant correlation between the severity of mucositis and the $T G F-\beta 1$ variant genotype. Given the results, the TGF- $\beta 1$ could not be considered an efficient prediction biomarker, but it may be useful as a biomarker for treatment follow-up.

Cytokines are also involved in RT-induced mucositis because they are released by disintegrating cells or by an immune reaction, resulting in the recruitment of inflammatory cells and in the development of toxicity $[42,62]$. Several researchers have investigated the variation in cytokine concentration, such as IL and TNF, in HNC patients undergoing CRT $[42,54,57,61]$. It was observed that while radiation dose increased, the levels of IL- 6 and IL-8 simultaneously increased, but only IL- 6 seemed to be related to severe mucositis $[42,54]$. Citrin et al. [42] found high levels of IL-10 in the saliva of patients with high-grade mucositis compared to those with low-grade mucositis. In contrast, Meirovitz et al. [54] did not find any significant changes in IL-10 levels. There were no significant changes in IL-1 levels [54, 57], but there was an increase in the expression of IL- $1 \beta$, which is a member of the interleukin-1 superfamily, and this increase was related to the radiation-induced OM [61].

The levels of TNF- $\alpha$ were also analyzed, and the results were again somewhat controversial. Two studies $[42,61]$ found increased levels of this cytokine during RT, while two other studies [54,57] showed decreased levels, and only Xanthinaki et al. [61] could find an association between TNF- $\alpha$ and OM. The results found that the cytokine levels were quite heterogeneous, probably because the cytokines 


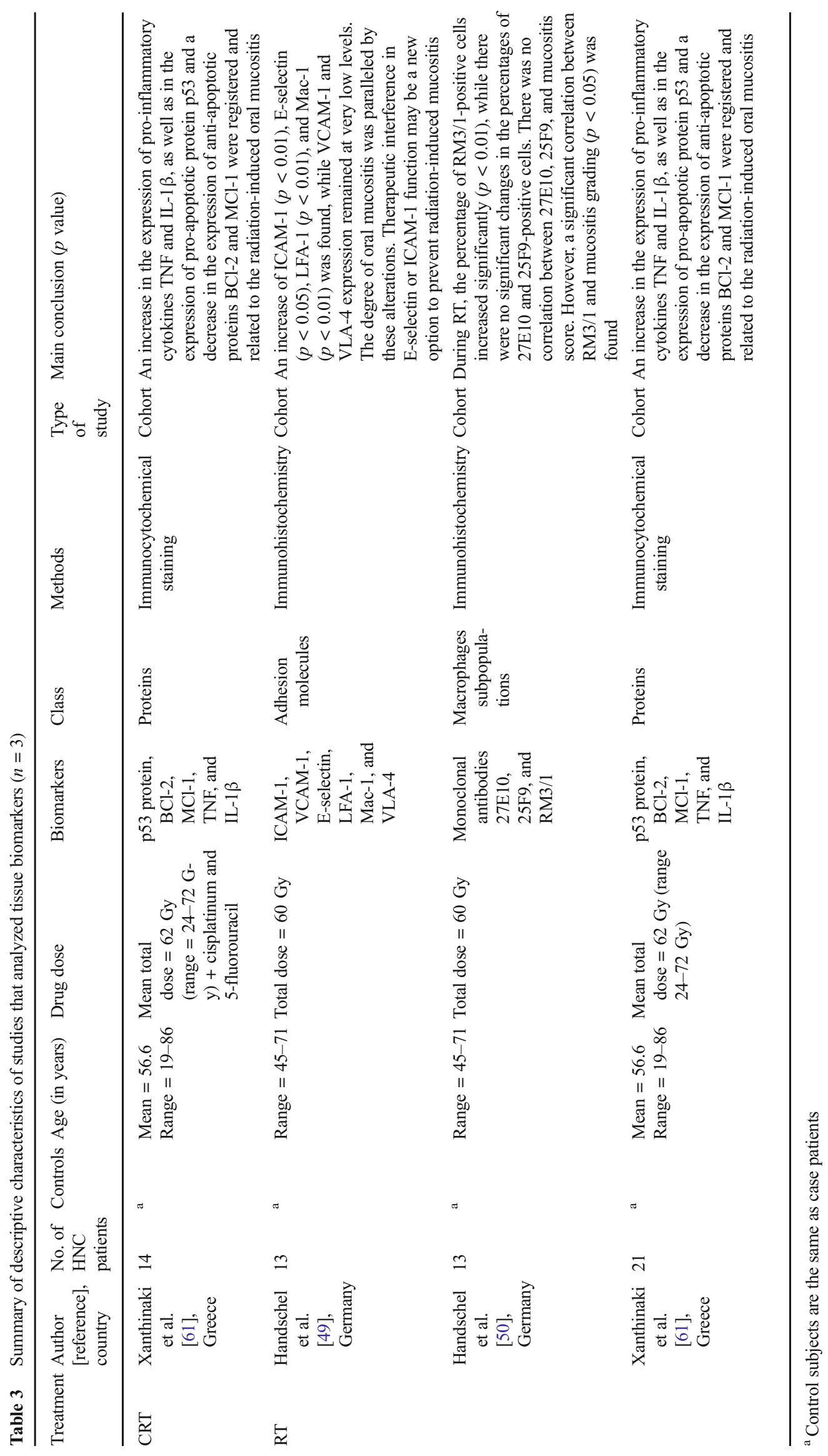


Table 4 Summary of the risk of bias assessment

\begin{tabular}{ll}
\hline Author & Risk of bias \\
\hline Bhattathiri et al. [38] & Moderate \\
Chen et al. [39] & Low \\
Chen et al. [40] & Low \\
Chethana et al. [41] & Moderate \\
Citrin et al. [42] & Low \\
Dumbrigue et al. [43] & Low \\
Ehrsson et al. [44] & Low \\
Epstein et al. [45] & Low \\
Epstein et al. [46] & Low \\
Fleckenstein et al. [47] & Low \\
Gonzalez et al. [48] & Low \\
Handschel et al. [49] & Low \\
Handschel et al. [50] & Low \\
Jehmlich et al. [11] & Moderate \\
Ki et al. [51] & Low \\
Li et al. [52] & Moderate \\
Lundberg et al. [53] & Low \\
Meirovitz et al. [54] & Low \\
Mohammed et al. [55] & Low \\
Pratesi et al. [56] & Moderate \\
Seyyednejad et al. [57] & Low \\
Venkatesh et al. [3] & Low \\
Vuotila et al. [58] & Low \\
Wardman et al. [59] & Low \\
Werbrouck et al. [60] & Moderate \\
Xanthinaki et al. [61] & Moderate \\
\hline & \\
\hline
\end{tabular}

${ }^{a}$ Assessed by the Meta-Analysis of Statistics Assessment and Review Instrument (MAStARI) [17] critical appraisal tools. Risk of bias was categorized as high when the study reached up to $49 \%$ score "yes," moderate when the study reached 50 to $69 \%$ score "yes," and low when the study reached more than $70 \%$ score "yes"

were analyzed in different fluids and the concentration may vary from saliva to serum. In this way, future studies with larger sample size could provide a definitive answer if cytokines can be effective in predicting an adverse response to RT [54].

Besides the predictor effect of growth factors and cytokines, there are evidences that these substances may be useful in preventing and treating OM. Palifermin, a keratinocyte growth factor (KGF), is already recommended to prevent oral mucositis in patients with hematological malignancies receiving high-dose CT and total body irradiation (TBI) [62]. A systematic review by Raber-Durlacher et al. [63] aimed to define evidence-based clinical practice guidelines for the use of cytokine and growth factor agents to prevent and treat mucositis. Sixty-seven studies were included in the review, assessing KGF, EGF, TGF- $\beta$, IL-11, granulocyte-macrophage colony-stimulating factor (GM-CSF), and granulocyte colony-stimulating factor (G-CSF). Due to insufficient and conflicting evidence, they could not provide a guideline for the use of none of these growth factors and cytokines for the prevention or treatment of $\mathrm{OM}$ in $\mathrm{HNC}$ patients.

Acute-phase inflammatory markers are also used as biomarkers to predict the risk for patients developing $\mathrm{OM}$ as a consequence of cancer treatment. CRP is one of these markers, and it contributes to body defense by neutralizing inflammatory agents and it can be easily measured as a quantitative marker of inflammatory activity [41]. CRP was reported to increase towards the end of RT [41, 44, 51, 55], and while $\mathrm{Ki}$ et al. [51] demonstrated a correlation between this increase and the progression of mucositis, Chethana et al. [41] could only observe this correlation during the initial weeks of treatment.

ESR is another important marker of the acute-phase inflammatory response, used to evaluate benign inflammatory conditions and neoplastic diseases [41, 51, 55]. An increase in ESR levels during cancer treatment was observed, followed by a decrease in concentration of this biomarker. This variation was related to the grading of mucositis, which also initially increased in severity and then decreased towards the end of treatment [41, 55]. Differently, Ki et al. [51] did not find any statistically significant relationship between ESR and mucositis grade. These data support that acute-phase inflammatory proteins may have the potential to act as objective mucositis markers, although their values vary significantly between patients [55].

General proteins, plasma antioxidants, apoptotic proteins, adhesion molecules, and cells were also used as biomarkers in the included studies. Although the number of studies was not so significant, the results were quite relevant. It was demonstrated that there was a correlation between OM grading and increasing levels of the proteins BPIFA-1 [48], ICAM-1, Eselectin, LFA-1, and Mac-1 [49], as well as the pro-apoptotic protein p53 [61] and RM3/1-positive cells [50]. Decreased expression of anti-apoptotic proteins $\mathrm{BCl}-2$ and $\mathrm{MCl}-1$ were also associated to radiation-induced OM [61]. The plasma antioxidant GSH was reported to be associated with $\mathrm{OM}$ and to have a radioprotective role [38], while Wardman et al. [59] could not find a correlation between mucositis severity and plasma antioxidants, including GSH. The meta-analysis showed an expression of $39.57 \%$ of the proteins BPIFA-1, BPIFA-2, LFA-1, Mac-1, VLA-4, p53, BCl-2, MCl-1, TNF, and IL- $1 \beta$ in the combined samples from the studies of González-Arriagada et al. [48], Handschel et al. [49], and Xanthinaki et al. [61]. The evidence was not strong, and this may be explained by the results that were not homogenous enough. Thus, further studies are still needed to confirm the efficacy of the use of inflammatory and non-inflammatory proteins as biomarkers of OM. 
Fig. 2 Frequency of biomarkers

Biomarkers: $\mathrm{BCl}-2 \mathrm{~B}$ cell or permeability-increasing protein family A, CRP C-reactive protein, $E G F$ epidermal growth factor, ESR erythrocyte sedimentation rate, $G S H$ glutathione, $I G F-1$ insulin growth factor $1, I G F B P-1$ insulin-like growth factor-binding protein $1, \mathrm{IL}$ interleukin, $\mathrm{MCl}-1$ myeloid cell leukemia 1, MCP-1 monocyte chemoattractant protein 1, MMP matrix metalloproteinase, $T N F$ - $\alpha$ tumor necrosis factor alpha, $T G F-\beta 1$ transforming growth factor beta 1 , $T P$ total protein, $V E G F$ vascular endothelial growth factor in the included studies.

lymphoma 2, BPIFA bactericidal

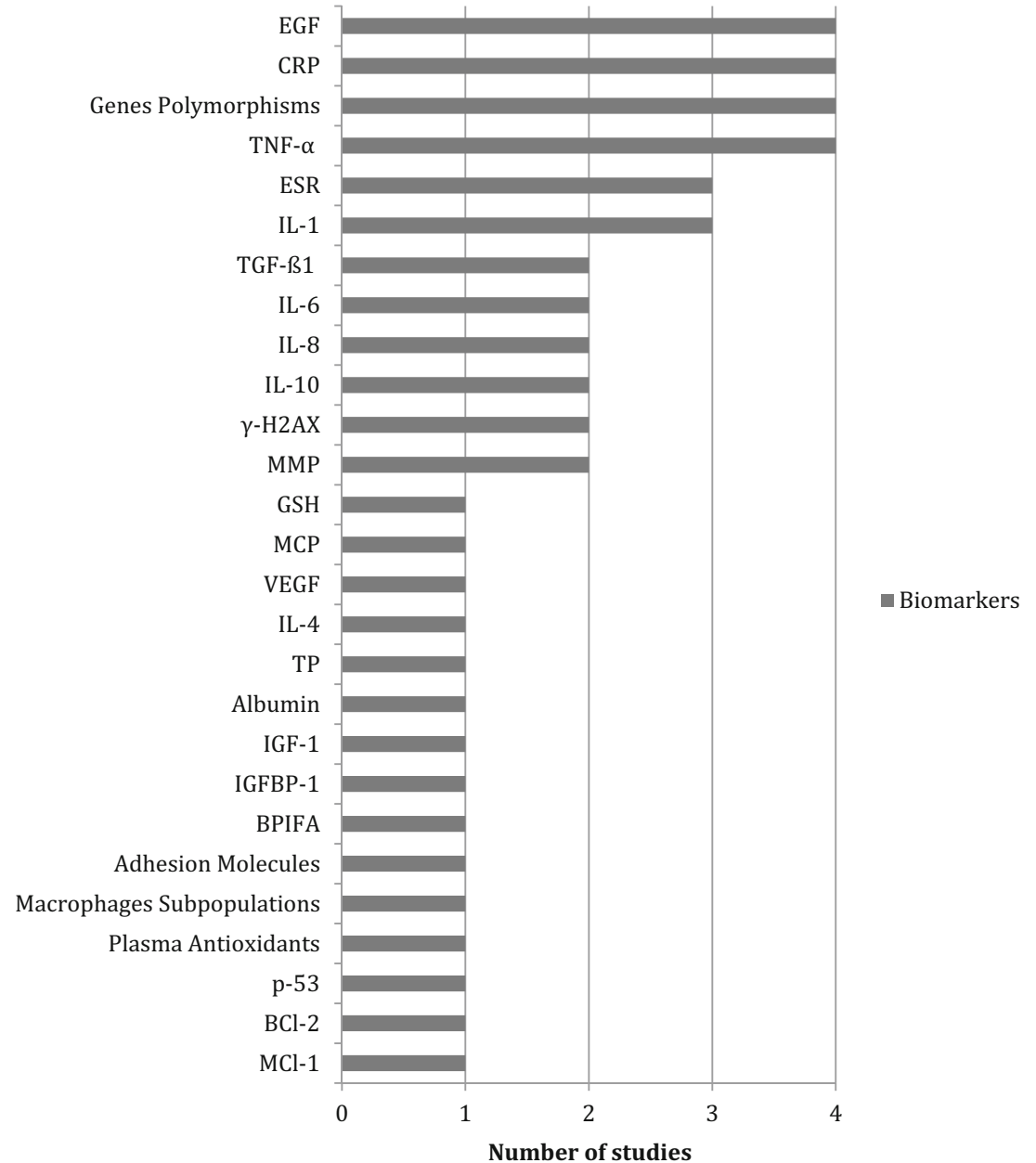

The extension of radiation-induced DNA damage and its repair are considered very relevant indicators of irradiation toxicity. The histone protein $\gamma-\mathrm{H} 2 \mathrm{AX}$, an essential factor in the repair process of damaged DNA, is immediately phosphorylated at sites of DNA double-strand breaks (DSBs), and its levels have been used to quantify the ability of cells
Fig. 3 Frequency of grouped biomarkers in the included studies

\section{Biomarkers}

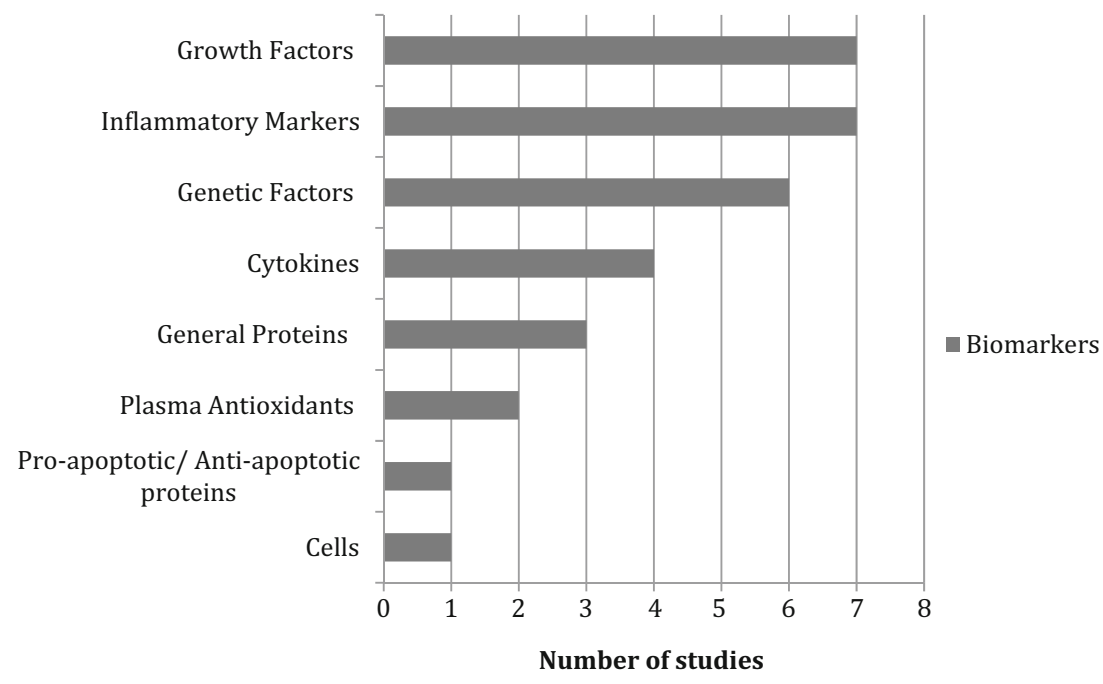


a

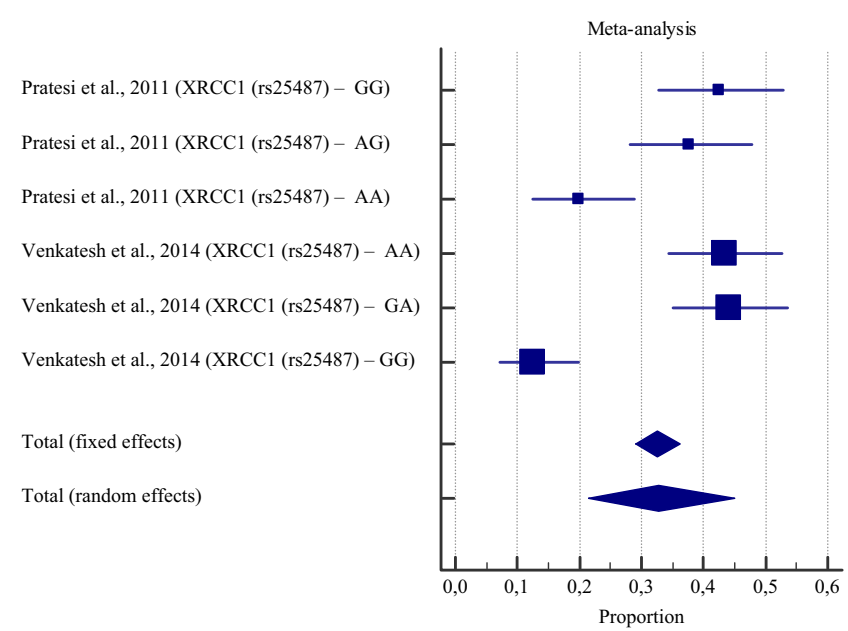

$\mathrm{c}$

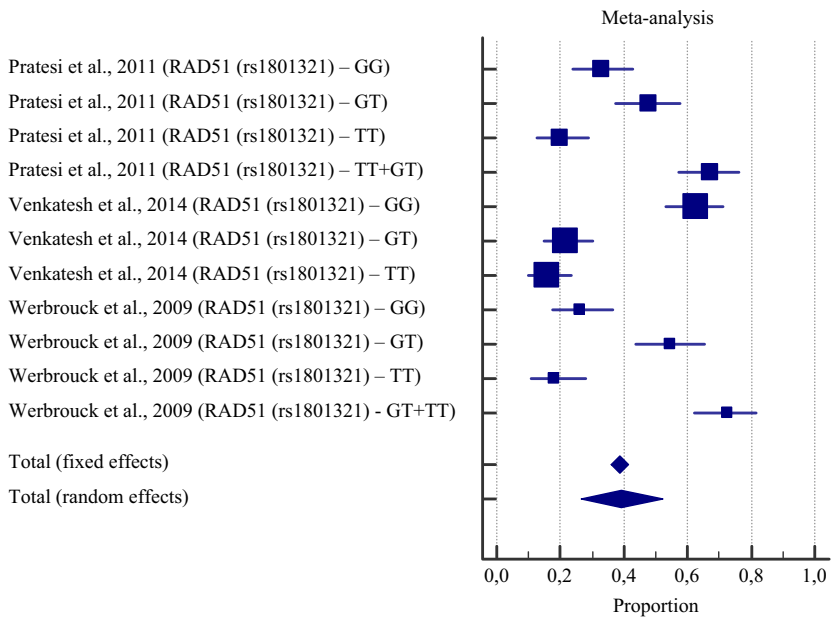

Fig. 4 Frequency of genetic polymorphisms and protein expression associated to OM risk. Results from two types of meta-analysis: fixed and random effects. a Forest plot for polymorphism in XRCC1 (rs25487)

to damage and repair DNA after irradiation [52]. A study by Li et al. [52] observed higher $\gamma-\mathrm{H} 2 \mathrm{AX}$ levels by the end of RT, but the increase in $\gamma$-H2AX expression was not statistically different between patients with mild OM and severe OM, although the patients with severe OM had a reduced capacity for DNA repair. In order to estimate sensitivity and specificity of the relative fluorescence of $\gamma-\mathrm{H} 2 \mathrm{AX}$ to predict the risk of OM during RT, they performed a receiver operating characteristic (ROC) analysis that indicated sensitivity and specificity of 100 and $53.3 \%$, respectively [51]. Another study also indicated that patients who developed mild mucositis had a total amount of DSB repair similar to patients who developed severe OM [47]. It was proven that the detection of $\gamma-\mathrm{H} 2 \mathrm{AX}$ induced by irradiation could be used to predict the incidence and severity of toxicities like OM, since it allows assessment of individual DSB repair after RT [47, 52]. b

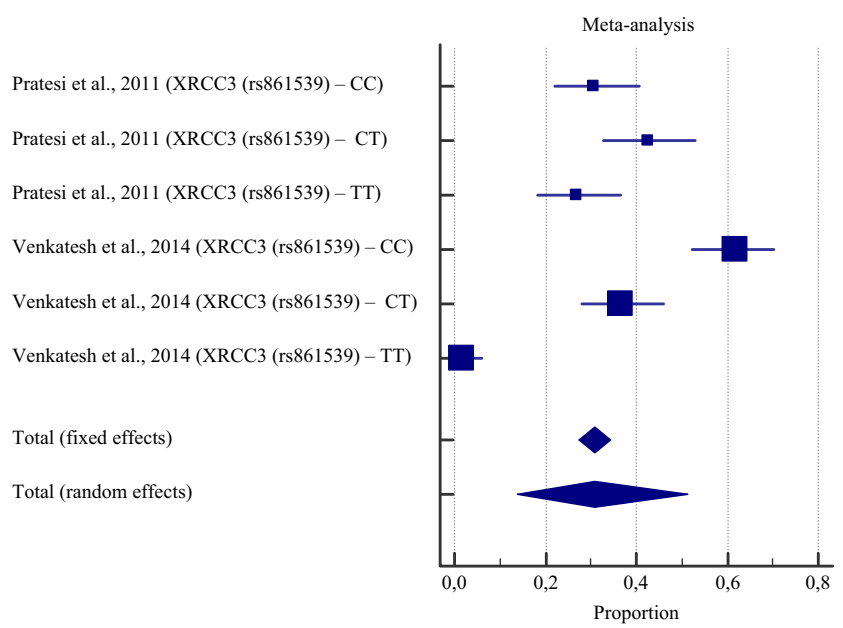

d

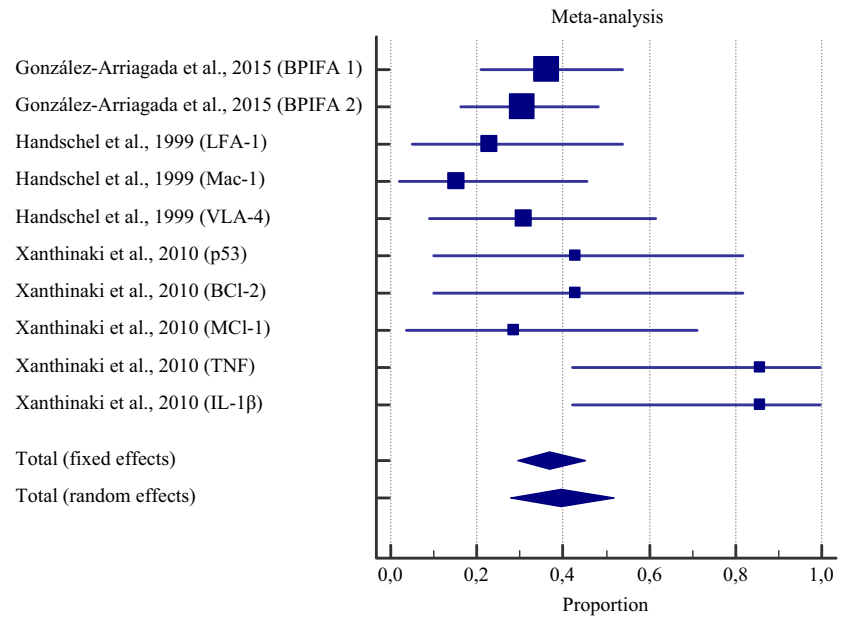

( sample $=663)$. b Forest plot for polymorphism in XRCC3 (rs861539) (sample $=663)$. $\mathbf{c}$ Forest plot for polymorphism in RAD51 (rs1801321) $($ sample $=1116)$. d Forest plot for protein expression $($ sample $=146)$

SNPs in DNA repair genes can modify their function and consequently interfere in the individual's capacity to repair damaged DNA; thus, variations in specific genes could be associated to the susceptibility of development of radiation toxicities [56]. It was demonstrated that polymorphisms in $\mathrm{XRCC1}$, XRCC3, and RAD51 genes were associated to an increased risk of developing toxicities related to RT, including severe OM [3, 56, 60]. Pratesi et al. [56] and Werbrouck et al. [60] tested the relationship between dose parameter and adverse radiation effects with the Mann-Whitney test. The following expression levels in the meta-analysis of polymorphisms: $32.66 \%$ (XRCC1), 31.00\% (XRCC3), and 39.16\% (RAD1) were found in the combined samples from the studies of Pratesi et al. [56], Venkatesh et al. [3], and Werbrouck et al. [60]. The evidence was not strong, and this may be explained by the results that were not homogenous enough. Despite the 
increasing number of studies regarding SNPs, the evidence is still not strong enough to suggest the use of these polymorphisms as biomarkers to predict tissue toxicity.

\section{Limitations}

Some methodological limitations of this review should be considered. First is the small number of patients included in the studies; however, it is important to notice that head and neck cancer is an uncommon cancer and a sample of approximately 20 patients in each study should be considered as representative. Second, many studies did not include in the analysis the outcomes of people who withdrew nor identified confounding factors, what increased the risk of bias of these studies. Lastly, the heterogeneity of biomarkers made it difficult to compare a significant amount of studies about the same marker.

\section{Conclusion}

This systematic review and meta-analysis demonstrates that biomarkers emerge as potential predictors for $\mathrm{OM}$ in $\mathrm{HNC}$ patients. Thus, dosing biomarkers related to mucositis before starting RT can identify radiosensitive individuals and allow these patients to have a customized treatment plan which might have less chances of interruption. Additionally, the biomarkers that have been proven to be more effective in predicting the risk of mucositis were CRP, ESR, and EGF. Although there is currently limited evidence to confirm the putative implementation of serum and salivary biomarkers to assess the correlation between them and the severity of mucositis, this current review provides new research directions. It is recommended that this new research be in the format of welldesigned experimental studies, following closely to research guidelines, and sensible to the most used and relevant biomarkers.

Acknowledgements The authors thank Mr. Wesam Ashour for providing language help.

\section{Compliance with ethical standards}

Funding This research did not receive any specific grant from funding agencies in the public, commercial, or not-for-profit sectors.

Conflict of interest The authors declare that they have no competing interests.

\section{References}

1. Shield KD, Ferlay J, Jemal A, Sankaranarayanan R et al (2017) The global incidence of lip, oral cavity, and pharyngeal cancers by subsite in 2012. CA Cancer J Clin 67(1):51-64

2. Cabrera AR, Yoo DS, Brizel DM (2013) Contemporary radiotherapy in head and neck cancer. Surg Oncol Clin N Am 22(3):579-598

3. Venkatesh GH, Manjunath VB, Mumbrekar KD et al (2014) Polymorphisms in radio-responsive genes and its association with acute toxicity among head and neck cancer patients. PLoS One 9(3):89079-89079

4. Vera-Llonch M, Oster G, Hagiwara M et al (2006) Oral mucositis in patients undergoing radiation treatment for head and neck carcinoma. Cancer 106(2):329-336

5. Scully C, Epstein J, Sonis S (2003) Oral mucositis: a challenging complication of radiotherapy, chemotherapy, and radiochemotherapy. Head Neck 25(12):1057-1070

6. Villa A, Sonis ST (2015) Mucositis: pathobiology and management. Curr Opin Oncol 27(3):159-164

7. Scully C, Epstein J, Sonis S (2004) Oral mucositis: a challenging complication of radiotherapy, chemotherapy, and radiochemotherapy. Part 2. Head Neck 26(1):77-84

8. Lalla RV, Bowen J, Barasch A et al (2014) MASCC/ISOO clinical practice guidelines for the management of mucositis secondary to cancer therapy. Cancer 120(10):1453-1461

9. Sonis ST (2009) Mucositis: the impact, biology and therapeutic opportunities of oral mucositis. Oral Oncol 45(12):1015-1020

10. Sonis ST, Elting LS, Keefe D et al (2004) Perspectives on cancer therapy-induced mucosal injury: pathogenesis, measurement, epidemiology, and consequences for patients. Cancer 100(9 Suppl):1995-2025

11. Jehmlich N, Stegmaier P, Golatowski C et al (2015) Differences in the whole saliva baseline proteome profile associated with development of oral mucositis in head and neck cancer patients undergoing radiotherapy. J Proteome 125:98-103

12. Strimbu K, Tavel JA (2010) What are biomarkers. Curr Opin HIV AIDS 5(6):463-466. doi:10.1097/COH.0b013e32833ed177

13. Patel S, Ahmed S (2015) Emerging field of metabolomics: big promise for cancer biomarker identification and drug discovery. J Pharm Biomed Anal 107:63-74

14. Moher D, Liberati A, Tetzlaff J, Altman DG, PRISMA Group (2010) Preferred reporting items for systematic reviews and metaanalyses: the PRISMA statement. Int J Surg 8:336-341

15. Normando AGCN, Rocha CL, de Toledo IP, et al. 2016 Biomarkers in the assessment of oral mucositis in head and neck cancer patients: a systematic review. PROSPERO: CRD42016037299. Available at: http://www.crd.york.ac.uk/PROSPERO/display record.asp?ID= CRD42016037299. Accessed September 20, 2016

16. National Comprehensive Cancer Network. 2016 NCCN clinical practice guidelines in oncology: head and neck cancers (version 2). [Accessed 20 Dec 2016]. Avaliable in: http:/www.nccn.org

17. The Joanna Briggs Institute. (2014). The Joanna Briggs Institute Reviewer's Manual 2014 Edition: Meta Analysis of Statistics Assessment and Review Instrument (MAStARI) critical appraisal tools Comparable cohort/ Case control studies. Adelaide, Australia: The Joanna Briggs Institute

18. Higgins JPT, Green S (editors). 2011. Cochrane Handbook for Systematic Reviews of Interventions version 5.1.0 [updated March 2011]. The Cochrane Collaboration, Available from www. handbook.cochrane.org.

19. Balshem $\mathrm{H}$ et al (2011) GRADE guidelines: 3 . Rating the quality of evidence. J Clin Epidemiol 64(4):401-406

20. Schünemann $\mathrm{H}$ et al. GRADE handbook for grading quality of evidence and strength of recommendations. Updated October 2013. The GRADE Working Group, 2013. Available from www. guidelinedevelopment.org/handbook 
21. Akmansu M, Unsal D, Bora $H$ et al (2005) Influence of locoregional radiation treatment on tumor necrosis factor- $\alpha$ and interleukin- 6 in the serum of patients with head and neck cancer. Cytokine 31(1):41-45

22. Ardito F, Giuliani M, Perrone D et al (2016) Expression of salivary biomarkers in patients with oral mucositis: evaluation by SELDITOF/MS. Oral Dis 22(3):209-219

23. Bonan PRF, Kaminagakura E, Pires FR et al (2007) Histomorphometry and immunohistochemical features of grade I (WHO) oral radiomucositis. Oral Dis 13(2):170-176

24. Bourton EC, Plowman PN, Smith D et al (2011) Prolonged expression of the $\gamma$-H2AX DNA repair biomarker correlates with excess acute and chronic toxicity from radiotherapy treatment. Int J Cancer 129(12):2928-2934

25. Christensen ME, Hansen HS, Poulsen SS et al (1996) Immunohistochemical and quantitative changes in salivary EGF, amylase and haptocorrin following radiotherapy for oral cancer. Acta Otolaryngol 116(1):137-143

26. Goutham HV, Mumbrekar KD, Vadhiraja BM et al (2012) DNA double-strand break analysis by $\gamma$-H2AX foci: a useful method for determining the overreactors to radiation-induced acute reactions among head-and-neck cancer patients. Int J Radiat Oncol Biol Phys 84(5):e607-e612

27. Hamilton S, Yoo J, Hammond A et al (2008) Microvascular changes in radiation-induced oral mucositis. J Otolaryngol Head Neck Surg 37(5):730-737

28. Henke M, Bechtold C, Momm F et al (2000) Blood hemoglobin level may affect radiosensitivity-preliminary results on acutely reacting normal tissues. Int J Radiat Oncol Biol Phys 48(2):339345

29. Ho AY, Atencio DP, Peters S et al (2006) Genetic predictors of adverse radiotherapy effects: the Gene-PARE project. Int J Radiat Oncol Biol Phys 65(3):646-655

30. Ikebe T, Yamasaki K, Takamune Y et al (2012) Reduced expression of nuclear factor $\mathrm{kB}$ in oral mucosa undergoing preoperative chemoradiotherapy. Oral Science International 9(2):33-37

31. Krause CE, Otieno BA, Bishop GW et al (2015) Ultrasensitive microfluidic array for serum pro-inflammatory cytokines and $\mathrm{C}$ reactive protein to assess oral mucositis risk in cancer patients. Anal Bioanal Chem 407(23):7239-7243

32. Oton-Leite AF, Silva GBL, Morais MO et al (2015) Effect of lowlevel laser therapy on chemoradiotherapy-induced oral mucositis and salivary inflammatory mediators in head and neck cancer patients. Lasers Surg Med 47(4):296-305

33. Popanda O, Marquardt JU, Chang-Claude J et al (2009) Genetic variation in normal tissue toxicity induced by ionizing radiation. Mutat Res 667(1-2):58-69

34. Sonis S, Haddad S, Posner M et al (2007) Gene expression changes in peripheral blood cells provide insight into the biological mechanisms associated with regimen-related toxicities in patients being treated for head and neck cancers. Oral Oncol 43(3):289-300

35. Verey F, Nexo E, Greenwood R et al (2011) Trefoil factor family peptides are increased in the saliva of children with mucositis. Clin Chem Lab Med 49(12):2051-2055

36. Werbrouck J, Duprez F, De Neve W et al (2011) Lack of a correlation between $\gamma \mathrm{H} 2 \mathrm{AX}$ foci kinetics in lymphocytes and the severity of acute normal tissue reactions during IMRT treatment for head and neck cancer. Int J Radiat Biol 87(1):46-56

37. Zou G, Lin X, Wu J et al (2012) Association of serum transforming growth factor-beta1 with radiation injury and survival of patients with early-stage nasopharyngeal carcinoma. Nan Fang Yi Ke Da Xue Xue Bao 32(8):1171-1174

38. Bhattathiri VN, Sreelekha TT, Sebastian P et al (1994) Influence of plasma GSH level on acute radiation mucositis of the oral cavity. Int J Radiat Oncol Biol Phys 29(2):383-386
39. Chen HW, Chang YC, Lai YL et al (2005) Change of plasma transforming growth factor-betal levels in nasopharyngeal carcinoma patients treated with concurrent chemo-radiotherapy. Jpn J Clin Oncol 35(8):427-432

40. Chen HW, Yang SF, Chang YC et al (2008) Epstein-Barr virus infection and plasma transforming growth factor-betal levels in head and neck cancers. Acta Otolaryngol 128(10):1145-1151

41. Chethana RPS, Madathil LP et al (2015) Quantitative analysis of acute phase proteins in post chemo-radiation mucositis. J Clin Diagn Res 9(10):ZC28-ZC31

42. Citrin DE, Hitchcock YJ, Chung EJ et al (2012) Determination of cytokine protein levels in oral secretions in patients undergoing radiotherapy for head and neck malignancies. Radiat Oncol 7:64

43. Dumbrigue HB, Sandow PL, Nguyen KHT et al (2000) Salivary epidermal growth factor levels decrease in patients receiving radiation therapy to the head and neck. Oral Surg Oral Med Oral Pathol Oral Radiol Endod 89(6):710-716

44. Ehrsson YT, Hellström PM, Brismar K et al (2010) Explorative study on the predictive value of systematic inflammatory and metabolic markers on weight loss in head and neck cancer patients undergoing radiotherapy. Support Care Cancer 18(11):1385-1391

45. Epstein JB, Emerton S, Guglietta A et al (1997) Assessment of epidermal growth factor in oral secretions of patients receiving radiation therapy for cancer. Oral Oncol 33(5):359-363

46. Epstein JB, Gorsky M, Guglietta A et al (2000) The correlation between epidermal growth factor levels in saliva and the severity of oral mucositis during oropharyngeal radiation therapy. Cancer 89(11):2258-2265

47. Fleckenstein J, Kühne M, Seegmüller K et al (2011) The impact of individual in vivo repair of DNA double-strand breaks on oral mucositis in adjuvant radiotherapy of head-and-neck cancer. Int J Radiat Oncol Biol Phys 81(5):1465-1472

48. Gonzalez-Arriagada WA, Ramos LMA, Silva AA et al (2015) Salivary BPIFA1 (SPLUNC1) and BPIFA2 (SPLUNC2 A) are modified by head and neck cancer radiotherapy. Oral Surg Oral Med Oral Pathol Oral Radiol 119(1):48-58

49. Handschel J, Prott FJ, Sunderkötter C et al (1999) Irradiation induces increase of adhesion molecules and accumulation of beta(2)integrin-expressing cells in humans. Int J Radiat Oncol Biol Phys 45(2):475-481

50. Handschel J, Sunderkötter C, Prott FJ et al (2001) Increase of RM3/ 1-positive macrophages in radiation-induced oral mucositis. $\mathrm{J}$ Pathol 193(2):242-247

51. Ki Y, Kim W, Nam J et al (2009) C-reactive protein levels and radiation-induced mucositis in patients with head-and-neck cancer. Int J Radiat Oncol Biol Phys 75(2):393-398

52. Li P, Du CR, Xu WC et al (2013) Correlation of dynamic changes in gamma-H2AX expression in peripheral blood lymphocytes from head and neck cancer patients with radiation-induced oral mucositis. Radiat Oncol 8:155

53. Lundberg M, Saarilahti K, Mäkitie AA et al (2010) TGF beta 1 genetic polymorphism is associated with survival in head and neck squamous cell carcinoma independent of the severity of chemoradiotherapy induced mucositis. Oral Oncol 46(5):369-372

54. Meirovitz A, Kuten M, Billan S et al (2010) Cytokines levels, severity of acute mucositis and the need of PEG tube installation during chemo-radiation for head and neck cancer-a prospective pilot study. Radiat Oncol 5:16

55. Mohammed FF, Poon I, Zhang L et al (2012) Acute-phase response reactants as objective biomarkers of radiation-induced mucositis in head and neck cancer. Head Neck 34(7):985-993

56. Pratesi N, Mangoni M, Mancini I et al (2011) Association between single nucleotide polymorphisms in the XRCC1 and RAD51 genes and clinical radiosensitivity in head and neck cancer. Radiother Oncol 99(3):356-361 
57. Seyyednejad F, Rezaee A, Haghi S et al (2012) Survey of preinflammation cytokines levels in radiotherapy-induced-mucositis. Pak J Biol Sci 15(22):1098-1101

58. Vuotila T, Ylikontiola L, Sorsa T et al (2002) The relationship between MMPs and $\mathrm{pH}$ in whole saliva of radiated head and neck cancer patients. J Oral Pathol Med 31(6):329-338

59. Wardman P, Folkes LK, Bentzen SM et al (2001) Influence of plasma glutathione levels on radiation mucositis. Int $\mathrm{J}$ Radiat Oncol Biol Phys 51(2):460-464

60. Werbrouck J, Ruyck KD, Duprez F et al (2009) Acute normal tissue reactions in head-and-neck cancer patients treated with IMRT: influence of dose and association with genetic polymorphisms in DNA DSB repair genes. Int J Radiat Oncol Biol Phys 73(4): $1187-1195$
61. Xanthinaki A, Nicolatou-Galitis O, Athanassiadou P et al (2008) Apoptotic and inflammation markers in oral mucositis in head and neck cancer patients receiving radiotherapy: preliminary report. Support Care Cancer 16(9):1025-1033

62. Von Bültzingsloöwen I, Brennan MT, Spijkervet FKL et al (2006) Growth factors and cytokines in the prevention and treatment of oral and gastrointestinal mucositis. Support Care Cancer 14(6): $519-527$

63. Raber-Durlacher JE, von Bültzingslöwen I, Logan RM et al (2013) Systematic review of cytokines and growth factors for the management of oral mucositis in cancer patients. Support Care Cancer 21: $343-355$ 